Prediction of lymphatic dissemination in endometrioid endometrial cancer : Comparison of three risk-stratification models in a single-institution cohort

\title{
Tuomi, Taru
}

2017-03

Tuomi , T , Pasanen , A , Leminen, A , Butzow , R \& Loukovaara , M 2017 , ' Prediction of lymphatic dissemination in endometrioid endometrial cancer : Comparison of three risk-stratification models in a single-institution cohort ' , Gynecologic Oncology , vol. 144 , no. 3 , pp. 510-514 . https://doi.org/10.1016/j.ygyno.2017.01.003

http://hdl.handle.net/10138/234619

https://doi.org/10.1016/j.ygyno.2017.01.003

publishedVersion

Downloaded from Helda, University of Helsinki institutional repository.

This is an electronic reprint of the original article.

This reprint may differ from the original in pagination and typographic detail.

Please cite the original version. 


\title{
Prediction of lymphatic dissemination in endometrioid endometrial cancer: Comparison of three risk-stratification models in a single-institution cohort
}

\author{
Taru Tuomi $^{\mathrm{a}, *}$, Annukka Pasanen ${ }^{\mathrm{b}}$, Arto Leminen ${ }^{\mathrm{a}}$, Ralf Bützow ${ }^{\mathrm{b}}$, Mikko Loukovaara ${ }^{\mathrm{a}}$ \\ a Department of Obstetrics and Gynecology, University of Helsinki and Helsinki University Hospital, Helsinki, Finland \\ ${ }^{\mathrm{b}}$ Department of Pathology, University of Helsinki and Helsinki University Hospital, Helsinki, Finland
}

\section{H I G H L I G H T S}

- The models were similarly accurate in predicting lymphatic dissemination in endometrial cancer

- The proportions of patients at low risk for lymphatic dissemination differed.

- The models also predicted disease specific survival in patients with stage I disease.

\section{A R T I C L E I N F O}

\section{Article history:}

Received 15 November 2016

Received in revised form 26 December 2016

Accepted 3 January 2017

Available online 7 January 2017

\section{Keywords:}

Disease specific survival

Endometrial cancer

External validation

Lymphatic dissemination

Risk-stratification

\begin{abstract}
A B S T R A C T
Objectives. To compare the performance characteristics of 3 risk-stratification models, referred to as Mayo Helsinki and Milwaukee models, in predicting lymphatic dissemination in endometrial cancer.

Methods. A total of 1052 patients with stage I-III endometrioid endometrial cancer were included in the study. The areas under curve were compared with the receiver operating characteristic curve area comparison test. Chi-square and Fisher exact test were used for comparing categorical variables. The Kaplan-Meier method and multivariable Cox regression models were used for survival analyses. The median follow-up time was 55 months (range 1-108).

Results. Areas under curve were $0.781,0.830$ and 0.829 for the Mayo, Helsinki ( $P=0.285$ vs. Mayo) and Milwaukee ( $P=0.292$ vs. Mayo) models, respectively, in predicting lymphatic dissemination. The rates of false negatives and false positives were similar for all models. The lymphadenectomy rate decreased in the order of Mayo model (71.5\%) > Helsinki model (62.4\%) > Milwaukee model (48.8\%). In patients with stage I cancer, disease specific survival was better for those who satisfied low-risk criteria according to any of the models. In patients with stage II-III cancer, this difference in survival was significant only for the Milwaukee model. Both lymphatic dissemination and high-risk tumor features as per the risk models were independent predictors of survival.

Conclusions. The studied models had a similar accuracy in predicting lymphatic dissemination in endometrial cancer. Lymphadenectomy rate was lowest for the Milwaukee model. Survival analyses suggest that variables included in the models predict patient outcome independently of tumor stage.
\end{abstract}

() 2017 Elsevier Inc. All rights reserved.

\section{Introduction}

The surgical treatment of patients with apparent early low-risk endometrial cancer consists of total hysterectomy with bilateral salpingo-oophorectomy. Pelvic-aortic lymphadenectomy is undertaken in high-risk patients for accurate staging, which improves prognostication and allows triage for tailored adjuvant treatment [1].

\footnotetext{
* Corresponding author at: Department of Obstetrics and Gynecology, University of Helsinki and Helsinki University Hospital, P.O. Box 140, 00029 HUS, Helsinki, Finland.

E-mail address: taru.tuomi@fimnet.fi (T. Tuomi).
}

The recognition of true low-risk endometrial cancers remains a challenge. In clinical practice, risk-stratification is mainly based on preoperative or intraoperative identification of the features of the primary tumor ("uterine risk factors"), sometimes combined with tumor markers in serum. A proper risk-stratification method is accurate, and concurrently associated with an acceptable lymphadenectomy rate. Ideally, it should also facilitate the prediction of patient survival.

In 2000, Mariani et al. introduced a risk-stratification schema that is now vastly utilized to define low-risk endometrial cancers [2]. According to these Mayo criteria, named after the Mayo Clinic, Rochester, $\mathrm{MN}$, the low-risk group is comprised of tumors with grade 1-2 
endometrioid histology, myometrial invasion $\leq 50 \%$, and diameter $\leq 2 \mathrm{~cm}$. Subsequently, Bogani et al. developed a 5-category risk-stratification system based on frozen section analysis where also noninvasive endometrioid carcinomas are included as low-risk cases, regardless of grade and size [3]. Recently, Cox Bauer et al. at the Aurora Sinai Medical Center and St. Luke's Medical Center, Milwaukee, WI, introduced a schema for endometrioid carcinomas that contains depth of myometrial invasion and tumor size as parameters for identifying patients at low risk for lymph node involvement, with low-risk criteria being satisfied when the depth of invasion is $\leq 33 \%$ and diameter $\leq 50 \mathrm{~mm}$, regardless of grade [4]. This schema was based on findings on final pathology but the variables were considered potential intraoperative predictors of lymphatic dissemination. Notably, the authors reported that these novel low-risk criteria allow for an additional $20 \%$ of patients to be spared surgical lymph node assessment, compared with the Mayo criteria. We have earlier demonstrated a combined preoperative and intraoperative scoring system for a prediction of stage IIIC-IV endometrial cancer [5]. Patients at low risk for an advanced disease were those with a normal platelet count and CA125 value, and grade 1-2 endometrioid carcinoma of $<3 \mathrm{~cm}$ in size according to preoperative histology and gross visual inspection.

To test the universal applicability of the risk models by Bogani et al. [3] and Cox Bauer et al. [4], we validated the findings of the original studies in our own cohort of endometrial cancer patients. The performance characteristics of the 2 models were compared with our own published model [5]. Further, we assessed the value of each model as a prognostic tool in endometrial cancer.

\section{Materials and methods}

Patients who underwent primary surgical treatment for stage I-IIIC endometrioid endometrial cancer at the Department of Obstetrics and Gynecology, Helsinki University Hospital, between January 1, 2007 and December 31, 2013 were included in this study $(n=1052)$. Carcinosarcomas $(n=16$ ) were included as high grade endometrioid carcinomas [6]. Indications for lymphadenectomy in our cohort have been reported earlier [7]. Briefly, the initial strategy was to perform pelvic lymphadenectomy routinely in all patients, and para-aortic lymphadenectomy selectively in patients considered to be at highest risk for lymph node metastasis. As of January 2012, routine pelvic lymphadenectomy was abandoned and the decision on lymphadenectomy was based on preoperative histology and assessment of local disease spread by magnetic resonance imaging. Pertinent patient characteristics and surgical and pathology data are shown in Table 1. Stage was determined according to the International Federation of Gynecology and Obstetrics (FIGO) guidelines revised in 2009 [8].

We compared 3 risk-stratification models for endometrioid endometrial cancer, referred to in the following as Mayo [3], Helsinki [5] and Milwaukee [4] models. For each model, the area under the receiver operating characteristic curve was built. Unlike the previously reported Mayo criteria [3,9], frozen section analysis was not available to us for review. Thus, women were identified as being at low risk for nodal metastasis based on modified Mayo criteria: grade 1-2 endometrioid histology, myometrial invasion $<50 \%$ and diameter $<2 \mathrm{~cm}$ on final pathology reports [10], and noninvasive cancers of any grade and size [3]. Findings on final pathology were also used for validation of the Helsinki and Milwaukee models. Similar to the Cox Bauer study [4], we restricted our analysis to stage I-IIIC cancers of the endometrioid type, although the original Helsinki model also considered stage IV and nonendometrioid carcinomas [5]. Risk schemas for the different models are detailed in Table 2. Patients with available data for all risk parameters considered in each model were included in the calculations ( $n=1045$ for the Mayo model, $n=881$ for the Helsinki model, $n=987$ for the Milwaukee model).

For the Helsinki model, pretreatment serum CA125 concentration was quantitated with the chemiluminescent microparticle
Table 1

Clinicopathologic data $(n=1052)$.

\begin{tabular}{|c|c|}
\hline Age (years) (mean $\pm \mathrm{SD})$ & $67.2 \pm 10.4$ \\
\hline Body mass index $\left(\mathrm{kg} / \mathrm{m}^{2}\right)(\text { mean } \pm \mathrm{SD})^{\mathrm{a}}$ & $28.7 \pm 6.3$ \\
\hline Pelvic lymphadenectomy (number of cases, percent) & $550(52.3 \%)$ \\
\hline Pelvic-aortic lymphadenectomy (number of cases, percent) & $134(12.7 \%)$ \\
\hline Lymph node yield, pelvic lymphadenectomy $(\text { mean } \pm S D)^{b}$ & $15.3 \pm 8.0$ \\
\hline Lymph node yield, pelvic-aortic lymphadenectomy $(\text { mean } \pm S D)^{c}$ & $26.1 \pm 9.9$ \\
\hline Laparoscopic hysterectomies (number of cases, percent) ${ }^{\mathrm{d}}$ & 859 (81.7\%) \\
\hline \multicolumn{2}{|l|}{ Adjuvant therapy (number of cases, percent) } \\
\hline Vaginal brachytherapy & $497(47.2 \%)$ \\
\hline Whole pelvic radiotherapy & $144(13.7 \%)$ \\
\hline Chemotherapy & $20(1.9 \%)$ \\
\hline Chemotherapy and vaginal brachytherapy & $27(2.6 \%)$ \\
\hline Chemotherapy and whole pelvic radiotherapy & $107(10.2 \%)$ \\
\hline \multicolumn{2}{|l|}{ Grade (number of cases, percent) } \\
\hline Grade 1 & $642(61.0 \%)$ \\
\hline Grade 2 & $264(25.1 \%)$ \\
\hline Grade $3^{\mathrm{e}}$ & $146(13.9 \%)$ \\
\hline \multicolumn{2}{|l|}{ FIGO 2009 stage (number of cases, percent) } \\
\hline IA & $653(62.1 \%)$ \\
\hline IB & $216(20.5 \%)$ \\
\hline II & $60(5.7 \%)$ \\
\hline IIIA & $43(4.1 \%)$ \\
\hline IIIB & $7(0.7 \%)$ \\
\hline IIIC1 & $51(4.8 \%)$ \\
\hline IIIC2 & $22(2.1 \%)$ \\
\hline $\begin{array}{l}\text { a Number of cases } 1051 \text { (body mass index missing for } 1 \text { patient). } \\
\text { b Number of cases } 542 \text { (lymph node yield missing for } 8 \text { patients). } \\
\text { c Number of cases } 132 \text { (lymph node yield missing for } 2 \text { patient). } \\
\text { d Traditional laparoscopic hysterectomies, } n=789 \text {; robotic hysterec } \\
\text { e Including } 16 \text { carcinosarcomas. }\end{array}$ & mies, $n=70$. \\
\hline
\end{tabular}

immunoassay on the Abbott Architect 2000i Analyzer (Abbott Diagnostics, Abbott Park, IL). The concentration was considered increased when $>35 \mathrm{U} / \mathrm{mL}$ [11]. Last pretreatment platelet count was analyzed by electrical impedance technology and flow cytometry. Thrombocytosis was defined as a platelet count $>360 \times 10^{9} / \mathrm{L}$ [12] .

The areas under curve were compared with the 2-tailed receiver operating characteristic curve area comparison test. Chi-square and Fisher

Table 2

Risk-stratification schemas.

\begin{tabular}{ll}
\hline Mayo & Low risk \\
TD $<2 \mathrm{~cm}$, grade 1 or $2, \mathrm{MI}<50 \%$ \\
$\mathrm{MI} 0 \%$, any TD or grade \\
Low-intermediate risk \\
TD $\geq 2$ cm or unknown, grade 1 or $2, \mathrm{MI}<50 \%$ \\
High-intermediate risk \\
Grade 1 or $2,50 \%<\mathrm{MI} \leq 66 \%$ \\
Grade 3 , MI $<50 \%$ \\
High risk \\
Grade 1 or 2 , MI $>66 \%$ \\
Grade 3 , MI $\geq 50 \%$ \\
Adnexal metastasis \\
Low risk \\
0 risk score points \\
Low-intermediate to high risk \\
$1-8$ risk score points \\
Low risk \\
TD $\leq 50$ mm, any grade, $\mathrm{MI} \leq 33 \%$ \\
Low-intermediate risk \\
TD $>50$ mm, any grade, $\mathrm{MI} \leq 33 \%$ \\
TD $\leq 50$ mm, any grade, $33 \%<\mathrm{MI} \leq 66 \%$ \\
TD $\leq 50$ mm, grade $1, \mathrm{MI}>66 \%$ \\
High-intermediate risk \\
TD $>50$ mm, grade $1,33 \%<\mathrm{MI} \leq 66 \%$ \\
TD $\leq 50$ mm, grade 2 or $3, \mathrm{MI}>66 \%$ \\
High risk \\
TD $>50$ mm, any grade, $\mathrm{MI}>66 \%$ \\
TD $>50$ mm, grade 2 or $3,33 \%<\mathrm{MI} \leq 66 \%$ \\
Milwaukee
\end{tabular}

MI, myometrial invasion; TD, tumor diameter.

a 1 point for thrombocytosis, 2 points for poor differentiation (grade 3), 2 points for TD $\geq 3 \mathrm{~cm}, 3$ points for CA125 $>35 \mathrm{U} / \mathrm{mL}$ 
Table 3

Areas under curve (AUC) for 3 models in predicting lymphatic dissemination in endometrioid endometrial cancer.

\begin{tabular}{|c|c|c|c|}
\hline & AUC $(95 \% \mathrm{CI})$ & Pvs. Mayo (2-tailed) & $P$ vs. Helsinki (2-tailed) \\
\hline \multicolumn{4}{|l|}{ All patients } \\
\hline Mayo $(n=1045)$ & $0.781(0.729-0.832)$ & & \\
\hline Helsinki $(n=881)$ & $0.830(0.790-0.871)$ & 0.285 & \\
\hline Milwaukee $(n=987)$ & $0.829(0.777-0.882)$ & 0.292 & 0.982 \\
\hline \multicolumn{4}{|c|}{ Patients who underwent lymphadenectomy } \\
\hline Mayo $(n=678)^{\mathrm{a}}$ & $0.761(0.703-0.818)$ & & \\
\hline Helsinki $(n=583)^{\mathrm{b}}$ & $0.815(0.767-0.863)$ & 0.280 & \\
\hline Milwaukee $(n=631)^{c}$ & $0.806(0.746-0.865)$ & 0.368 & 0.855 \\
\hline
\end{tabular}

a Pelvic lymphadenectomy, $n=546$; pelvic-aortic lymphadenectomy, $n=132$.

b Pelvic lymphadenectomy, $n=463$; pelvic-aortic lymphadenectomy, $n=120$.

c Pelvic lymphadenectomy, $n=504$; pelvic-aortic lymphadenectomy, $n=127$.

exact test were used for comparing categorical variables. Disease specific survival, defined as the time from date of surgery to death from endometrial cancer, was estimated using the Kaplan-Meier method. Differences between groups were compared using the log rank test. The joint effect of 2 factors on survival was assessed using multivariable Cox regression analysis. The median follow-up time was 55 months (range 1-108). Statistical significance was set at $P<0.05$. Data were analyzed using IBM SPSS version 22 software (IBM Corp., Armonk, NY).

\section{Results}

Areas under curve were $0.781,0.830$ and 0.829 for the Mayo, Helsinki and Milwaukee models, respectively, in predicting lymphatic dissemination in endometrioid endometrial cancer, indicating a fair to good discrimination power for all models (Table 3). Differences in areas under curve were not significant (Table 3). These findings retained after exclusion of patients who did not undergo pelvic or pelvic-aortic lymphadenectomy (Table 3).

Diagnostic indices in predicting lymphatic dissemination by the 3 models are shown in Table 4. With cut-off set at low-intermediate risk for the Mayo and Milwaukee models and 1 risk score point for the Helsinki model, sensitivity was highest for the Helsinki model and specificity highest for the Milwaukee model. These findings remained unaltered when the analyses were restricted to patients who underwent pelvic or pelvic-aortic lymphadenectomy (Table 4). Despite the observed differences in sensitivities and specificities, the rates of false negatives and false positives were similar for all models (Table 5).

Lymphadenectomy rates corresponded to the proportion of patients who did not satisfy low-risk criteria according to the Mayo and Milwaukee criteria, or had $\geq 1$ risk score points according to the Helsinki criteria. The expected lymphadenectomy rate was $71.5 \%$ (747/1045) for the Mayo model, 62.4\% (550/881) for the Helsinki model (odds ratio 0.66 [95\% confidence interval $0.55-0.80$ ] vs. Mayo; $P<0.0001$ ), and $48.8 \%$ (482/987) for the Milwaukee model (odds ratio 0.57 [95\% confidence interval 0.48-0.69] vs. Helsinki; $P<0.0001$ ).

In patients with stage I cancer, disease specific survival was better for those who satisfied low-risk criteria according to any of the models (Fig. 1A-C). This result also pertained to a subgroup of patients who underwent surgical staging (Supplemental Fig. S1). In patients with stage II-III cancer, the difference in survival was significant only for the Milwaukee model $(P=0.034$ ) (Fig. 1D-F). In Cox multivariable disease specific survival analyses, both lymphatic dissemination (stage IIIC) and high-risk tumor features as per the risk models were recognized as independent predictors of survival (Table 6).

\section{Discussion}

The surgical treatment of patients with endometrial cancer is nowadays tailored based on individual risk-assessment. Those at low risk for extrauterine disease do not benefit from lymphadenectomy and are generally treated with hysterectomy and bilateral salpingo-oophorectomy alone. This practice decreases the risk for intraoperative complications, such as vessel and nerve damage, and protects patients from the development of long-term sequelae of lymphadenectomy, including lower-extremity lymphedema and pelvic lymphatic cysts.

Numerous algorithms have been developed for the recognition of endometrial cancer patients who are at low risk for lymphatic spread. While the performance characteristics of the Mayo model have been verified prospectively [10] and in a population-based analysis [13], those of the other algorithms are usually based on single-institution experience. Here, we wanted to compare a modified Mayo model with Helsinki and Milwaukee models, both of which were recently introduced for the improvement of risk-assessment of endometrial cancer patients. The potential benefit of the Helsinki model lies in its clinical applicability, as its use does not require frozen section analysis or imaging studies [5]. The Milwaukee model, on the other hand, was reported to markedly reduce the number of lymphadenectomies performed on low-risk patients [4].

In a population-based analysis of patients with stage I or IIIC endometrioid endometrial cancer, $21.1 \%$ of the patients satisfied lowrisk Mayo criteria, defined as grade 1-2 endometrioid histology, myometrial invasion $<50 \%$, and tumor diameter $\leq 2 \mathrm{~cm} \mathrm{[13].} \mathrm{The} \mathrm{higher}$ proportion (28.5\%) of low-risk patients in our study is partly explained by the fact that we adhered to Mayo criteria that include noninvasive cancers of any grade and size as low-risk cancers [3]. Further, stage IIIIIB cancers were included in our calculations. Without considering

Table 4

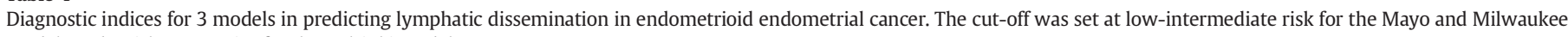
models and 1 risk score point for the Helsinki model.

\begin{tabular}{|c|c|c|c|c|}
\hline & $\begin{array}{l}\text { Sensitivity } \\
(95 \% \mathrm{CI})\end{array}$ & $\begin{array}{l}\text { Specificity } \\
(95 \% \mathrm{CI})\end{array}$ & Positive predictive value (95\% CI) & Negative predictive value $(95 \% \mathrm{CI})$ \\
\hline \multicolumn{5}{|l|}{ All patients } \\
\hline Mayo $(n=1045)$ & $94.4 \%(86.2 \%-98.4 \%)$ & $30.2 \%(27.3 \%-33.2 \%)$ & $9.0 \%(7.0 \%-11.3 \%)$ & $98.7 \%(96.6 \%-99.6 \%)$ \\
\hline Helsinki $(n=881)$ & $100 \%(94.5 \%-100 \%)$ & $40.6 \%(37.2 \%-44.0 \%)$ & $11.8 \%(9.2 \%-14.8 \%)$ & $100 \%(98.9 \%-100 \%)$ \\
\hline Milwaukee $(n=987)$ & $91.0 \%(81.5 \%-96.6 \%)$ & $54.2 \%(51.0 \%-57.5 \%)$ & $12.7 \%(9.8 \%-16.0 \%)$ & $98.8 \%(97.4 \%-99.6 \%)$ \\
\hline \multicolumn{5}{|c|}{ Patients who underwent lymphadenectomy } \\
\hline Mayo $(n=678)$ & $93.8 \%(84.8 \%-98.3 \%)$ & $28.0 \%(24.5 \%-31.8 \%)$ & $12.0 \%(9.3 \%-15.1 \%)$ & $97.7 \%(94.3 \%-99.4 \%)$ \\
\hline Helsinki $(n=583)$ & $100 \%(93.8 \%-100 \%)$ & $37.1 \%(33.0 \%-41.4 \%)$ & $15.0 \%(11.6 \%-18.9 \%)$ & $100 \%(98.1 \%-100 \%)$ \\
\hline Milwaukee $(n=631)$ & $90.0 \%(79.5 \%-96.2 \%)$ & $50.6 \%(46.4 \%-54.8 \%)$ & $16.1 \%(12.3 \%-20.4 \%)$ & $98.0 \%(95.6 \%-99.3 \%)$ \\
\hline
\end{tabular}


Table 5

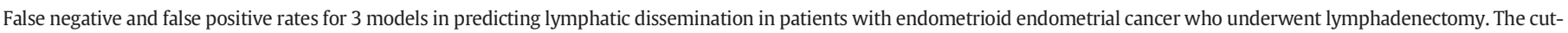
off was set at low-intermediate risk for the Mayo and Milwaukee models and 1 risk score point for the Helsinki model.

\begin{tabular}{|c|c|c|c|c|c|c|}
\hline & False negative rate & $\begin{array}{l}\text { P vs. Mayo } \\
\text { OR (95\% CI) }\end{array}$ & $\begin{array}{l}\text { P vs. Helsinki } \\
\text { OR }(95 \% \mathrm{CI})\end{array}$ & False positive rate & $\begin{array}{l}\text { P vs. Mayo } \\
\text { OR (95\% CI) }\end{array}$ & $\begin{array}{l}\text { P vs. Helsinki } \\
\text { OR }(95 \% \mathrm{CI})\end{array}$ \\
\hline Mayo & $4 / 176(2.3 \%)$ & & & $442 / 502(88.0 \%)$ & & \\
\hline Helsinki & 0/195 (0\%) & $\begin{array}{l}0.050 \\
0.98(0.96-1.0)\end{array}$ & & $330 / 388(85.1 \%)$ & $\begin{array}{l}0.191 \\
0.77(0.52-1.1)\end{array}$ & \\
\hline Milwaukee & $6 / 295(2.0 \%)$ & $\begin{array}{l}1.0 \\
0.89(0.25-3.2)\end{array}$ & $\begin{array}{l}0.086 \\
1.0(1.0-1.0)\end{array}$ & $282 / 336(83.9 \%)$ & $\begin{array}{l}0.088 \\
0.71(0.48-1.1)\end{array}$ & $\begin{array}{l}0.677 \\
0.92(0.61-1.4)\end{array}$ \\
\hline
\end{tabular}

noninvasive cancers as a separate entity in the schema and with the exclusion of stage II-IIIB cancers, the proportion of low-risk patients was $25.8 \%$ (242/937) in our sample, which is more comparable to that reported earlier [13]. The risk for lymph node metastasis in surgically staged low-risk Mayo patients was reported as $1.4 \%$ [13], which is in the same range as in our low-risk patients who underwent lymphadenectomy (2.3\%).

In the study by Cox Bauer et al. [4], 38.8\% of the patients had a lowrisk cancer according to the Milwaukee rules (51.2\% in our study), and their risk for lymph node involvement was $0 \%$ ( $2.0 \%$ in our study). The higher proportion of low-risk patients in our study did not diminish the accuracy of the model, as its area under curve in predicting lymph node metastasis was 0.829 , i.e. fairly similar to that in the discovery cohort (0.837) [4]. As expected, the findings concerning the Helsinki model were similar to those in our earlier report [5], although the inclusion criteria were slightly different from the original and tumor grade was based on findings in the hysterectomy specimens instead of preoperative endometrial samples.

All of the tested models had similar areas under curve in predicting lymphatic dissemination in endometrioid endometrial cancer. Moreover, the rates of false negatives and false positives were similar for all models. The Milwaukee model was associated with the lowest lymphadenectomy rate, a beneficial feature when avoidance of unnecessary lymphadenectomies is considered a quality measure. However, as both the Cox Bauer study [4] and the current analysis were based on findings on final pathology, institutions that consider implementation of the Milwaukee schema in clinical practice will need to validate it with techniques that will be used for the assessment of the depth of myometrial invasion and tumor size. Frozen section analysis has been shown to be reliable in assessing the features of the primary tumor, although a large sample flow may be a prerequisite for the development of an appropriate pathology expertise in a frozen section practice [14, 15]. The reliability of imaging techniques may generally not be high enough in assessing the local spread of endometrial cancer; in a multicenter prospective study, the sensitivities of transvaginal ultrasound and magnetic resonance imaging in detecting deep $(\geq 50 \%)$ myometrial invasion were $71 \%$ and $87 \%$, respectively [16]. Tumor diameter can be easily measured on fresh tissue intraoperatively and is unchanged on final pathology [17].

Koskas et al. performed an external validation of the Helsinki model and found that it had a low discrimination power in predicting stage IIIC-IV disease in their sample of 374 women (area under curve 0.68) [18]. It should be noted that the sample was somewhat anomalous regarding the proportion of type 2 and advanced cancers, as $22.7 \%$ were serous or clear cell carcinomas and $17.4 \%$ were stage IIIC-IV cancers. In our original study [5], the proportion of serous and clear cell carcinomas was $8.2 \%$ which corresponds to the common estimate of $10 \%$ as their proportion [19]. The proportion of stage IIIC-IV cancers was $11.1 \%$ in our study [5] which is fairly similar to the proportion of $7.9 \%$ in the Surveillance, Epidemiology, and End Results database [20]. These differences may explain why all risk parameters in the discovery cohort [5] were not significant predictors of an advanced disease in the validation cohort [18]. The accuracy of the scoring system might have been better if the aberrations in the validation cohort had been taken into account
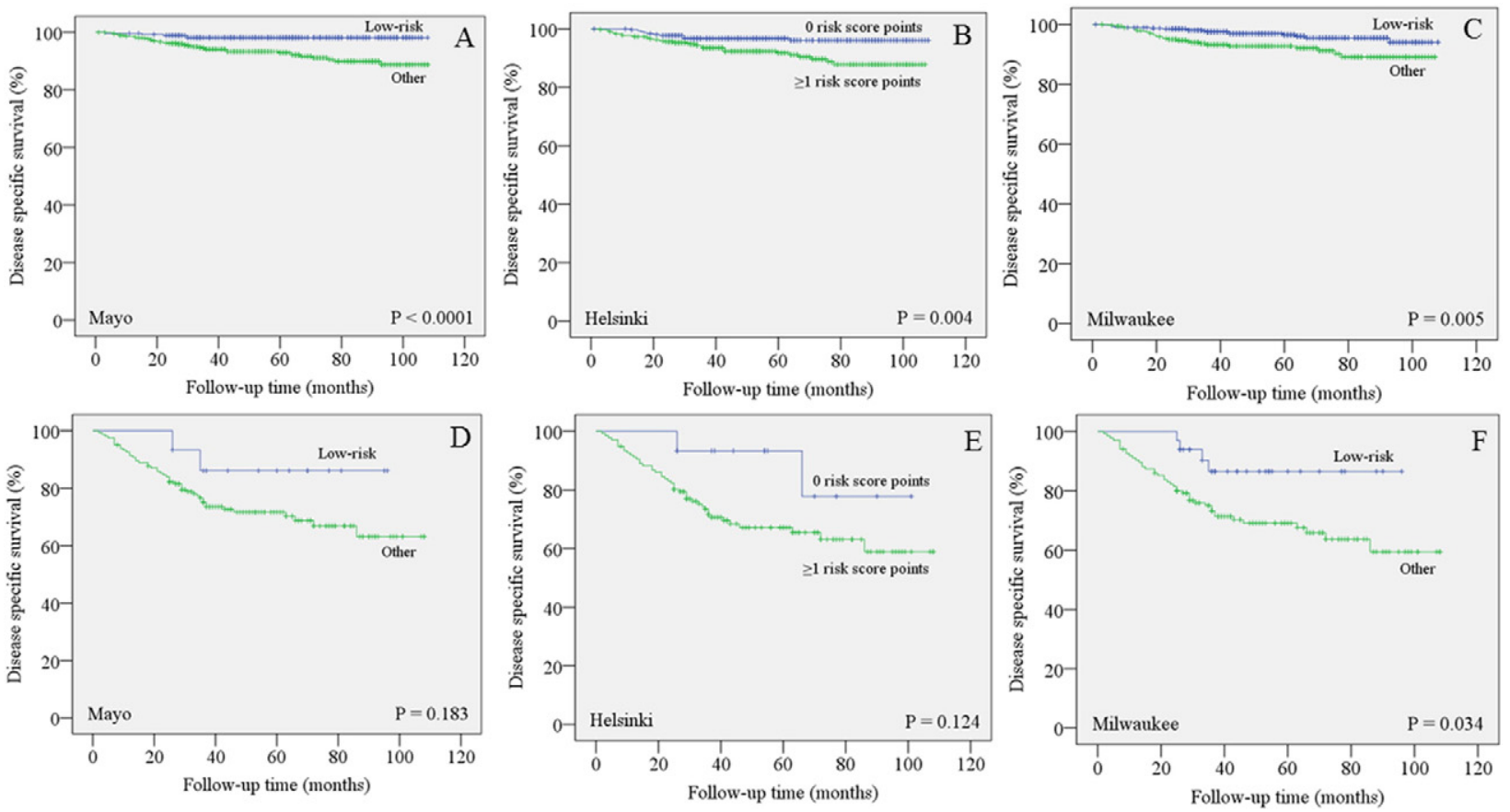

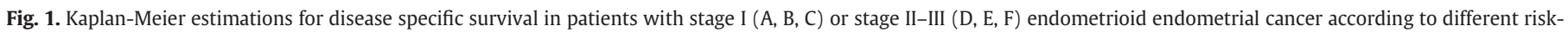
stratification models. 


\section{Table 6}

Cox multivariable disease specific survival analyses in patients with endometrioid endometrial cancer.

\begin{tabular}{lll}
\hline & HR $(95 \% \mathrm{CI})$ & $P$ \\
\hline Stage & & \\
$\quad$ I-IIIB $(n=974)$ & 1 & $<0.0001$ \\
$\quad$ IIIC $(n=71)$ & $4.9(3.1-7.8)$ & \\
Mayo model & & \\
$\quad$ Low risk $(n=298)$ & 1 & $<0.0001$ \\
$\quad$ Low-intermediate to high risk $(n=747)$ & $4.7(2.2-10)$ & \\
Stage & & \\
$\quad$ I-IIIB $(n=816)$ & 1 & $<0.0001$ \\
$\quad$ IIIC $(n=65)$ & $5.0(3.1-7.9)$ & \\
Helsinki model & 1 & $<0.0001$ \\
$\quad 0$ risk score points $(n=331)$ & $3.0(1.6-5.5)$ & \\
$\quad$ 1-8 risk score points $(n=550)$ & & \\
Stage & 1 & $<0.0001$ \\
$\quad$ I-IIIB $(n=920)$ & $4.8(3.0-7.7)$ & \\
$\quad$ IIIC $(n=67)$ & & 1 \\
Milwaukee model & & \\
$\quad$ Low risk $(n=505)$ & $2.9(1.7-4.8)$ & \\
$\quad$ Low-intermediate to high risk $(n=482)$ &
\end{tabular}

by calculating the risk score points as described [5], instead of using original points as such.

Patients with stage I cancer not satisfying low-risk criteria according to any of the models had a poorer disease specific survival than those with stage I low-risk cancer, which can be explained by the notion that uterine risk factors predict the outcome of endometrial cancer patients independently of nodal status $[21,22]$. This emphasizes the need for a 2-step risk-stratification in endometrial cancer. In the first phase, the risk for lymphatic dissemination is assessed preoperatively and/or intraoperatively, and this information is used to determine the need for lymphadenectomy. In the second phase, the need for adjuvant therapy is primarily determined by final histology and stage. However, because some patients with a local endometrioid cancer will have a poorer than expected outcome based on stage alone, additional variables will need to be considered in their risk-assessment. Currently, these mainly include uterine risk factors and patient age, but molecular classification of the tumors will probably allow refinement of adjuvant therapy decisions in the future [23]. The need for an accurate and comprehensive risk-assessment is emphasized by the fact that the proportion of patients with stage I cancer and poor outcome may be even higher than that of patients with stage IIIC cancer. Of the patients with stage I cancer in our study population, 10.0\% (87/869) had a disease relapse during follow-up, a higher proportion than the proportion of patients who had primary lymph node metastasis (6.9\%) (Table 1$)$.

In summary, the studied models had similar accuracies in predicting lymphatic dissemination in endometrioid endometrial cancer. Our findings regarding the Mayo model and the Milwaukee model are in agreement with earlier studies $[4,13]$, which demonstrates external validity. The low lymphadenectomy rate associated with the Milwaukee model may encourage its validation as a preoperative or intraoperative riskstratification schema by incorporating imaging or frozen section in the assessment of the depth of myometrial invasion. Patients with a nonlow-risk local endometrial cancer cannot be assured of a similarly good outcome as patients with a low-risk local disease.

Supplementary data to this article can be found online at http://dx. doi.org/10.1016/j.ygyno.2017.01.003.

\section{Conflict of interest statement}

The authors declare that there are no conflicts of interest associated with this manuscript.

\section{References}

[1] SGO Clinical Practice Endometrial Cancer Working Group, W.M. Burke, J. Orr, M. Leitao, E. Salom, P. Gehrig, A.B. Olawaiye, et al., Endometrial cancer: a review and current management strategies: part I, Gynecol. Oncol. 134 (2014) 385-392.

[2] A. Mariani, M.J. Webb, G.L. Keeney, M.G. Haddock, G. Calori, K.C. Podratz, Low-risk corpus cancer: is lymphadenectomy or radiotherapy necessary? Am. J. Obstet. Gynecol. 182 (2000) 1506-1519.

[3] G. Bogani, S.C. Dowdy, W.A. Cliby, F. Ghezzi, D. Rossetti, A. Mariani, Role of pelvic and para-aortic lymphadenectomy in endometrial cancer: current evidence, J. Obstet. Gynaecol. Res. 40 (2014) 301-311.

[4] C.M. Cox Bauer, D.M. Greer, J.J.F. Kram, S.A. Kamelle, Tumor diameter as a predictor of lymphatic dissemination in endometrioid endometrial cancer, Gynecol. Oncol. 141 (2016) 199-205.

[5] T. Tuomi, A. Pasanen, A. Luomaranta, A. Leminen, R. Bützow, M. Loukovaara, Riskstratification of endometrial carcinomas revisited: a combined preoperative and intraoperative scoring system for a reliable prediction of an advanced disease, Gynecol. Oncol. 137 (2015) 23-27.

[6] L.A. Cantrell, S.V. Blank, L.R. Duska, Uterine carcinosarcoma: a review of the literature, Gynecol. Oncol. 137 (2015) 581-588.

[7] A. Luomaranta, R. Bützow, A.-R. Pauna, A. Leminen, M. Loukovaara, Combined use of endometrial sample and magnetic resonance imaging in the preoperative risk-stratification of endometrial carcinomas, Acta Obstet. Gynecol. Scand. 94 (2015) 95-101.

[8] S. Pecorelli, Revised FIGO staging for carcinoma of the vulva, cervix, and endometrium, Int. J. Gynaecol. Obstet. 105 (2009) 103-104.

[9] A. Mariani, S.C. Dowdy, W.A. Cliby, B.S. Gostout, M.B. Jones, T.O. Wilson, et al., Prospective assessment of lymphatic dissemination in endometrial cancer: a paradigm shift in surgical staging, Gynecol. Oncol. 109 (2008) 11-18.

[10] M.R. Milam, J. Java, J.L. Walker, D.S. Metzinger, L.P. Parker, R.L. Coleman, et al., Nodal metastasis risk in endometrioid endometrial cancer, Obstet. Gynecol. 119 (2012) 286-292.

[11] R.C. Bast Jr., M. Feeney, H. Lazarus, L.M. Nadler, R.B. Colvin, R.C. Knapp, Reactivity of a monoclonal antibody with human ovarian carcinoma, J. Clin. Invest. 68 (1981) 1331-1337.

[12] V. Kairisto, P. Grönroos, M. Loikkanen, E.-R. Savolainen, K. Punnonen, M. Syrjälä et al., New Finnish reference limits for the basic blood count, Suom. Laakaril. 58 (2003) 5147-5153.

[13] R. Vargas, J.A. Rauh-Hain, J. Clemmer, R.M. Clark, A. Goodman, W.B. Growdon, et al., Tumor size, depth of invasion, and histologic grade as prognostic factors of lymph node involvement in endometrial cancer: a SEER analysis, Gynecol. Oncol. 133 (2014) 216-220.

[14] S. Kumar, F. Medeiros, S.C. Dowdy, G.L. Keeney, J.N. Bakkum-Gamez, K.C. Podratz, et al., A prospective assessment of the reliability of frozen section to direct intraoperative decision making in endometrial cancer, Gynecol. Oncol. 127 (2012) 525-531.

[15] J.M. Stephan, J. Hansen, M. Samuelson, M. McDonald, Y. Chin, D. Bender, et al., Intraoperative frozen section results reliably predict final pathology in endometrial cancer, Gynecol. Oncol. 133 (2014) 499-505.

[16] S.L. Antonsen, L.N. Jensen, A. Loft, A.K. Berthelsen, J. Costa, A. Tabor, et al., MRI, PET $\mathrm{CT}$ and ultrasound in the preoperative staging of endometrial cancer - a multicenter prospective comparative study, Gynecol. Oncol. 128 (2013) 300-308.

[17] M.M. AlHilli, K.C. Podratz, S.C. Dowdy, J.N. Bakkum-Gamez, A.L. Weaver, M.E. McGree, et al., Preoperative biopsy and intraoperative tumor diameter predict lymph node dissemination in endometrial cancer, Gynecol. Oncol. 128 (2013) 294-299.

[18] M. Koskas, J. Uzan, A. Vanderstraeten, I. Vergote, F. Amant, External validation of non-imaging models for predicting distant metastasis in patients with endometrial cancer, Gynecol. Oncol. 142 (2016) 83-88.

[19] F.D. Cirisano, S.J. Robboy, R.K. Dodge, R.C. Bentley, H.R. Krigman, I.S. Synan, et al., Epidemiologic and surgicopathologic findings of papillary serous and clear cell endometrial cancers when compared to endometrioid carcinoma, Gynecol. Oncol. 74 (1999) 385-394.

[20] E.W. Cooke, L. Pappas, D.K. Gaffney, Does the revised International Federation of Gynecology and Obstetrics staging system for endometrial cancer lead to increased discrimination in patient outcomes? Cancer 117 (2011) 4231-4237.

[21] J.S. Kwon, F. Qiu, R. Saskin, M.S. Carey, Are uterine risk factors more important than nodal status in predicting survival in endometrial cancer? Obstet. Gynecol. 114 (2009) 736-743.

[22] N.I. Barrena Medel, T.J. Herzog, I. Deutsch, W.M. Burke, X. Sun, S.N. Lewin, J.D. Wright, Comparison of the prognostic significance of uterine factors and nodal status for endometrial cancer, Am. J. Obstet. Gynecol. 204 (2011) 248.e1-248.e7.

[23] The Cancer Genome Atlas Research Network, C. Kandoth, N. Schultz, A.D. Cherniack R. Akbani, Y. Liu, H. Shen, et al., Integrated genomic characterization of endometrial carcinoma, Nature 497 (2013) 67-73. 